\title{
Sum Outage-Rate Maximization for MIMO Interference Channels
}

\author{
Juho Park, Donggun Kim and Younchul Sung ${ }^{\dagger}$ \\ Dept. of Electrical Engineering \\ KAIST \\ Daejeon, Korea 305-701 \\ Email: \{jhp@,dg.kim@ and ysung@ee.\}kaist.ac.kr
}

\begin{abstract}
In this paper, the weighted sum outage-rate maximization for time-invariant multiple-input multiple-output (MIMO) interference channels is considered. The cumulative distribution function (CDF) of outage events in MIMO interference channels is characterized under the assumption of Gaussian distribution for channel uncertainty. Based on the derived expression for the outage probability an iterative beam design algorithm for maximizing the weighted sum outage-rate under outage constraints is newly proposed. Numerical results show that the proposed algorithm shows good sum outage-rate performance.
\end{abstract}

\section{INTRODUCTION}

Interference management is a long-standing problem in wireless communications. There have been many interference managing schemes based on different approaches. Recently, interference alignment (IA) was proposed for multi-user interference channels, and it was shown that IA achieved maximum degrees of freedom at high signal-to-noise ratio (SNR) [1]. Since then there has been active research for IA and MIMO interference channels. While achievable degrees of freedom and feasibility of IA are characterized from informationtheoretical perspectives [2], [3], there also have been extensive efforts in developing good beam design algorithms for MIMO interference channels from practical perspectives. Available beam design algorithms include the iterative interference alignment (IIA), max-SINR algorithm [4], sum-rate maximizing algorithms [5], [6] alternating maximization [7], iterative least squares algorithm [8] only to name a few. However, most of these works assume perfect channel state information at transmitters (CSIT) and receivers, and such an assumption is prohibitive in real wireless communication systems. Only partial or imperfect channel information such as channel mean and error covariance or channel estimate at certain time instance is available; perfect CSIT is usually unavailable in practical wireless communication systems due to channel estimation error, limited feedback, and other difficulties [9]. There are several works that consider the beam design problem for MIMO interference channels with imperfect CSIT [10], [11]. In [10], the authors considered a non-probabilistic channel error model in which the channel estimation error

$\dagger$ This research was supported by Basic Science Research Program through the National Research Foundation of Korea (NRF) funded by the Ministry of Education, Science and Technology (2010-0021269). is characterized by a Frobenius norm constraint, and applied semi-definite relaxation to obtain optimal beams. In [11], on the other hand, the channel error is modelled as independent Gaussian random variable and beams are designed to minimize mean square error (MSE).

In this paper we consider the rate outage due to channel uncertainty* and the problem of sum outage-rate maximization in MIMO interference channels. This formulation is practically meaningful since an outage probability is assigned to each user and the supportable rate with the given outage probability is maximized in practical communication setups. Here, we assume that the transmitters have imperfect channel estimates and the channel estimation error or uncertainty is Gaussian distributed. Under the assumption, closed-form expressions for the exact cumulative distribution function (CDF) of outage for MIMO interference channels are derived for various setups, and an iterative beam design method for maximizing the weighted sum outage-rate is proposed based on the obtained outage probability expressions.

\section{A. Notation}

We will make use of standard notational conventions. Vectors and matrices are written in boldface with matrices in capitals. All vectors are column vectors. For matrix A, $\mathbf{A}^{H}$ and $\mathbf{A}(i, j)$ indicates the Hermitian transpose and the element of row $i$ and column $j$ of $\mathbf{A}$, respectively. $\mathbf{I}_{n}$ stands for the identity matrix of size $n$. vec(A) denotes the vector composed of the columns of $\mathbf{A}$, and $\operatorname{diag}\left(d_{1}, \cdots, d_{n}\right)$ means a diagonal matrix with diagonal elements $d_{1}, \cdots, d_{n}$. For vector a, $\|\mathbf{a}\|$ represents the 2-norm of $\mathbf{a} . \mathbf{x} \sim \mathcal{N}(\boldsymbol{\mu}, \boldsymbol{\Sigma})$ means that random vector $\mathbf{x}$ is complex Gaussian distributed with mean $\boldsymbol{\mu}$ and covariance matrix $\boldsymbol{\Sigma} . \mathbb{E}\{\cdot\}$ denotes the expectation and $\mathcal{K}=\{1,2, \cdots, K\} .\left[a_{i}\right]_{i \in \mathbf{A}}$ denotes a vector consisting of elements $a_{i}, i \in \mathbf{A}$.

\section{Data Model and Problem Formulation}

In this paper we consider a $K$-user time-invariant MIMO interference channel. Each transmitter is assumed to transmit $d$ independent streams to the corresponding receiver. Due to the interference from other users, the received signal at receiver $k$ at time $t$ is given by

\footnotetext{
${ }^{*}$ With perfect channel knowledge at transmitters and receivers, outages do not occur. The channel, transmit and receive beams support the rate calculated based on perfect CSIT.
} 


$$
\mathbf{y}_{k}(t)=\mathbf{H}_{k k}(t) \mathbf{V}_{k}(t) \mathbf{s}_{k}(t)+\sum_{l \neq k} \mathbf{H}_{k l}(t) \mathbf{V}_{l}(t) \mathbf{s}_{l}(t)+\mathbf{n}_{k}(t)
$$

where $\mathbf{H}_{k l}$ is the $M \times N$ channel matrix from transmitter $l$ to receiver $k$, and $\mathbf{V}_{l}=\left[\mathbf{v}_{l}^{(1)}, \cdots, \mathbf{v}_{l}^{(d)}\right]$ and $\mathbf{s}_{l}$ are the $N \times d$ transmit beamforming matrix and $d \times 1$ symbol vector at transmitter $l$, respectively. (The time index is omitted for notational simplicity.) We assume that the transmit symbol vector $\mathbf{s}_{l}$ is drawn from zero-mean Gaussian distribution with unit power, the beam vector has unit norm $\left\|\mathbf{v}_{l}^{(m)}\right\|=1$, and the additive noise $\mathbf{n}_{k} \sim \mathcal{N}\left(0, \sigma^{2} \mathbf{I}\right)$. Thus, the signal-to-noise ratio (SNR) is defined as $\mathrm{SNR}=\frac{1}{\sigma^{2}}$. At the receiver, the receive filter $\mathbf{u}_{k}^{(m)}$ with norm one at receiver $k$ projects $\mathbf{y}_{k}$ to obtain the desired signal of stream $m$, i.e.,

$$
\begin{aligned}
\hat{s}_{k}^{(m)} & =\left(\mathbf{u}_{k}^{(m)}\right)^{H} \mathbf{y}_{k}, \\
& =\left(\mathbf{u}_{k}^{(m)}\right)^{H} \mathbf{H}_{k k} \mathbf{V}_{k} \mathbf{s}_{k}+\left(\mathbf{u}_{k}^{(m)}\right)^{H}\left(\sum_{l \neq k} \mathbf{H}_{k l} \mathbf{V}_{l} \mathbf{s}_{l}+\mathbf{n}_{k}\right) .
\end{aligned}
$$

We model the imperfect CSIT as

$$
\mathbf{H}_{k l}=\hat{\mathbf{H}}_{k l}+\mathbf{E}_{k l},
$$

where $\hat{\mathbf{H}}_{k l}$ is the CSIT available at the transmitters and receivers, and $\mathbf{E}_{k l}$ is the channel uncertainty or error between the true and available channel information. We further assume that the elements of $\mathbf{E}_{k l}$ are independent and identically Gaussian distributed, i.e., $\mathbf{E}_{k l}(i, j) \stackrel{\text { i.i.d. }}{\sim} \mathcal{N}\left(0, \sigma_{h}^{2}\right)$. Such a channel estimation model is widely used for various MIMO systems e.g. [12]. Note here that in this formulation the true channel conditioned on the available channel information is modelled as Gaussian random matrix with mean $\hat{\mathbf{H}}_{k l}$ and covariance matrix of $\mathbb{E}\left\{\operatorname{vec}\left(\mathbf{H}_{k l}-\hat{\mathbf{H}}_{k l}\right) \operatorname{vec}\left(\mathbf{H}_{k l}-\hat{\mathbf{H}}_{k l}\right)^{H}\right\}=\sigma_{h}^{2} \mathbf{I}_{M N}$ for all $k, l \in \mathcal{K}$. Hereafter, we will use $\hat{\mathcal{H}}$ to denote the collection of channel information available to the users.

Due to the channel uncertainty, the receivers experience outages, and the $\epsilon$-outage-capacity region is defined as follows.

Definition 1: ( $\epsilon$-outage-capacity region) Given channel estimate $\hat{\mathcal{H}}$ and transmit/receive beamforming matrices $\left\{\mathbf{V}_{k}, \mathbf{U}_{k}\right\}$ based on $\hat{\mathcal{H}}, \mathcal{R}_{\epsilon}\left(\mathbf{V}_{k}, \mathbf{U}_{k}, \hat{\mathcal{H}}\right)$ is defined as a set of $K d$ rate-tuples $\left(R_{1}^{(1)}, R_{1}^{(2)}, \cdots, R_{1}^{(d)}, \cdots, R_{K}^{(d)}\right)$ such that

$$
\operatorname{Pr}\left\{\log \left(1+\left.\operatorname{SINR}_{k}^{(m)}\right|_{\hat{\mathcal{H}}}\right) \leq R_{k}^{(m)}\right\} \leq \epsilon
$$

for each $k$ and $m$, where $\operatorname{SINR}_{k}^{(m)}$ is the signalto-interference-and-noise ratio (SINR) for stream $m$ of transmitter-receiver pair $k$. Then, the $\epsilon$-outage-capacity region is defined by the union of $\mathcal{R}_{\epsilon}\left(\mathbf{V}_{k}, \mathbf{U}_{k}, \hat{\mathcal{H}}\right)$ over all transmit and receive beamforming vectors:

$$
\begin{gathered}
\mathcal{R}_{\epsilon}(\hat{\mathcal{H}}) \triangleq \bigcup_{\left\{\mathbf{V}_{k}, \mathbf{U}_{k}\right\}}\left\{\left(R_{1}^{(1)}, R_{1}^{(2)}, \cdots, R_{1}^{(d)}, \cdots, R_{K}^{(d)}\right) \mid\right. \\
\left.\operatorname{Pr}\left\{\log \left(1+\left.\operatorname{SINR}_{k}^{(m)}\right|_{\hat{\mathcal{H}}}\right) \leq R_{k}^{(m)}\right\} \leq \epsilon, k \in \mathcal{K}, m=1, \cdots, d\right\} .
\end{gathered}
$$

In this paper, we consider the following beam design problem to maximize the weighted sum outage-rate:

$$
\begin{array}{ll} 
& \left.\max _{\mathbf{V}_{k}, \mathbf{U}_{k}} \sum_{k=1}^{K} \sum_{m=1}^{d} w_{k}^{(m)} R_{k}^{(m)}\right|_{\hat{\mathcal{H}}} \\
\text { s.t. } & \operatorname{Pr}\left\{\log \left(1+\left.\operatorname{SINR}_{k}^{(m)}\right|_{\hat{\mathcal{H}}}\right) \leq R_{k}^{(m)}\right\} \leq \epsilon, \\
& \left\|\mathbf{u}_{k}^{(m)}\right\|=\left\|\mathbf{v}_{k}^{(m)}\right\|=1, \quad \forall k \in \mathcal{K}, \forall m .
\end{array}
$$

Here the weighting coefficients $\left\{w_{k}^{(m)}\right\}$ are nonnegative scalars. Note that the solution to the above problem is one of the points in $\mathcal{R}_{\epsilon}(\hat{\mathcal{H}})$ and further it is one of the Pareto optimal points of $\mathcal{R}_{\epsilon}(\hat{\mathcal{H}})$. Although there have been several works on the outage-rate region and beam design strategies to achieve a Pareto optimal point of the outage region in more tractable MISO interference channels [13], [14], there is not much available work in more complicated MIMO interference channels as we consider here. To do this we need to derive the probability distribution of outage in MIMO interference channels.

First, note that the outage event in (6) is equivalent to (8). The SINR at each stream is a random variable due to the channel uncertainty. By rearranging terms in (8), the outage event can be expressed as a function of random variables as

$$
\begin{aligned}
& \mathbf{Y}_{k}^{(m)} \triangleq \sum_{l \neq k} \sum_{n=1}^{d} \mathbf{X}_{k l}^{(m n) H} \mathbf{X}_{k l}^{(m n)}+\sum_{n \neq m} \mathbf{X}_{k k}^{(m n) H} \mathbf{X}_{k k}^{(m n)} \\
& +\mathbf{X}_{k k}^{(m m) H} \mathbf{X}_{k k}^{(m m)} \geq \frac{\left|\left(\mathbf{u}_{k}^{(m)}\right)^{H} \hat{\mathbf{H}}_{k k} \mathbf{v}_{k}^{(m)}\right|^{2}}{2^{R_{k}^{(m)}}-1}-\sigma^{2}=: y
\end{aligned}
$$

where

$$
\mathbf{X}_{k l}^{(m n)} \triangleq \begin{cases}\left(\mathbf{u}_{k}^{(l)}\right)^{H} \mathbf{E}_{k k} \mathbf{v}_{k}^{(l)}, & l=k, n=m, \\ \left(\mathbf{u}_{k}^{(m)}\right)^{H}\left(\hat{\mathbf{H}}_{k l}+\mathbf{E}_{k l}\right) \mathbf{v}_{l}^{(n)}, & \text { otherwise. }\end{cases}
$$

It can easily be shown that $\mathbf{X}_{k l}^{(m n)}$ is a complex Gaussian random variable with mean $\mathbf{u}_{k}^{(m) H} \hat{\mathbf{H}}_{k l} \mathbf{v}_{l}^{(n)}$ (except $\mathbb{E}\left[\mathbf{X}_{k k}^{(m m)}\right]=$ 0 ) and variance $\sigma_{h}^{2}$. Hence the random variable $\mathbf{Y}_{k}^{(m)}$ is a general quadratic form of non-central Gaussian random variables. The computation of outage probability will be presented in the next section.

\section{Computation of Outage Probability}

Let $\mathbf{X} \sim \mathcal{N}(\boldsymbol{\mu}, \mathbf{R})$ and $\mathbf{R}=\mathbf{U} \boldsymbol{\Lambda} \mathbf{U}^{H}$ be the eigendecomposition of $\mathbf{R}$. Suppose $\overline{\mathbf{X}} \sim \mathcal{N}(\mathbf{0}, \mathbf{I})$. Then,

$$
\mathbf{U} \boldsymbol{\Lambda}^{\frac{1}{2}}\left(\overline{\mathbf{X}}+\boldsymbol{\Lambda}^{-\frac{1}{2}} \mathbf{U}^{H} \boldsymbol{\mu}\right)=\mathbf{X}
$$

Hence, the quadratic form of $\mathbf{X}$ is expressed with $\overline{\mathbf{X}}$ as

$$
\begin{aligned}
& \mathbf{X}^{H} \overline{\mathbf{Q}} \mathbf{X} \\
& =\left[\mathbf{U} \boldsymbol{\Lambda}^{\frac{1}{2}}\left(\overline{\mathbf{X}}+\boldsymbol{\Lambda}^{-\frac{1}{2}} \mathbf{U}^{H} \boldsymbol{\mu}\right)\right]^{H} \overline{\mathbf{Q}}\left[\mathbf{U} \boldsymbol{\Lambda}^{\frac{1}{2}}\left(\overline{\mathbf{X}}+\boldsymbol{\Lambda}^{-\frac{1}{2}} \mathbf{U}^{H} \boldsymbol{\mu}\right)\right] \\
& =(\overline{\mathbf{X}}+\underbrace{\boldsymbol{\Lambda}^{-\frac{1}{2}} \mathbf{U}^{H} \boldsymbol{\mu}}_{\left(\triangleq_{\mathbf{q}}\right)})^{H} \underbrace{\boldsymbol{\Lambda}^{\frac{H}{2}} \mathbf{U}^{H} \overline{\mathbf{Q}} \mathbf{U} \boldsymbol{\Lambda}^{\frac{1}{2}}}_{\left(\triangleq_{\mathbf{Q}}\right)}\left(\overline{\mathbf{X}}+\boldsymbol{\Lambda}^{-\frac{1}{2}} \mathbf{U}^{H} \boldsymbol{\mu}\right) \\
& =(\overline{\mathbf{X}}+\mathbf{q})^{H} \mathbf{Q}(\overline{\mathbf{X}}+\mathbf{q})=\|\overline{\mathbf{X}}+\mathbf{q}\|_{\mathbf{Q}}^{2} .
\end{aligned}
$$




$$
\begin{aligned}
& \left.\operatorname{SINR}_{k}^{(m)}\right|_{\hat{\mathcal{H}}}= \\
& \frac{\left|\left(\mathbf{u}_{k}^{(m)}\right)^{H} \hat{\mathbf{H}}_{k k} \mathbf{v}_{k}^{(m)}\right|^{2}}{\sum_{n \neq m}\left|\left(\mathbf{u}_{k}^{(m)}\right)^{H}\left(\hat{\mathbf{H}}_{k k}+\mathbf{E}_{k k}\right) \mathbf{v}_{k}^{(n)}\right|^{2}+\sum_{l \neq k} \sum_{n=1}^{d}\left|\left(\mathbf{u}_{k}^{(m)}\right)^{H}\left(\hat{\mathbf{H}}_{k l}+\mathbf{E}_{k l}\right) \mathbf{v}_{l}^{(n)}\right|^{2}+\left|\left(\mathbf{u}_{k}^{(m)}\right)^{H} \mathbf{E}_{k k} \mathbf{v}_{k}^{(m)}\right|^{2}+\sigma^{2}} \leq 2^{R_{k}^{(m)}}-1 .
\end{aligned}
$$

The CDF of quadratic forms of Gaussian random variables has been studied before [15]-[17]. Especially, the CDF of (11) is given in [17] as

$$
\operatorname{Pr}\left\{\mathbf{X}^{H} \overline{\mathbf{Q}} \mathbf{X} \leq y\right\}=\frac{1}{2 \pi} \int_{-\infty}^{\infty} \frac{e^{y(j w+\beta)}}{j w+\beta} \frac{e^{-c}}{\operatorname{det}(\mathbf{I}+(j w+\beta) \mathbf{Q})} d w
$$

where $c=\mathbf{q}^{H}\left(\mathbf{I}+\frac{1}{j w+\beta} \mathbf{Q}^{-1}\right)^{-1} \mathbf{q}$ and $\beta \geq 0 .^{\dagger}$ From now on, we will derive closed-form expressions of the outage probability for several important setups using similar techniques to [16]. We start with the case that perfect CSIT is available on the desired link whereas imperfect CSIT is available on the interfering links. Such a scenario is reasonable in inter-cell collaborating downlink cellular systems in which each basestation can learn the channel of the desired link by feedback from its serving terminal station and data exchange among basestations is limited.

Theorem 1: When perfect channel information on the desired link is available and $d=1$, the probability of outage at user $k$ is given by

$$
\begin{aligned}
& \operatorname{Pr}\{\text { outage }\}=-\frac{1}{\left(\sigma_{h}^{2}\right)^{K-1}} e^{-\left(\frac{y}{\sigma_{h}^{2}}+\|\mathbf{q}\|^{2}\right)} \\
& \quad \times \sum_{n=K-2}^{\infty} \frac{1}{n !} g^{(n)}(0) \frac{1}{(n-K+2) !}\left(\frac{\|\mathbf{q}\|^{2}}{\sigma_{h}^{2}}\right)^{n-K+2}
\end{aligned}
$$

where $\mathbf{q} \triangleq \frac{1}{\sigma_{h}}\left[\mathbb{E}\left\{\mathbf{X}_{k l}\right\}\right]_{l \neq k}=\frac{1}{\sigma_{h}}\left[\mathbf{u}_{k}^{H} \hat{\mathbf{H}}_{k l} \mathbf{v}_{l}\right]_{l \neq k}$ and $g(s)=$ $\frac{e^{y s}}{s-1 / \sigma_{h}^{2}}$.

Proof: Here the superscript is omitted since it is always 1 in case of $d=1$. The assumption of users' having perfect information on the desired link implies that $\mathbf{E}_{k k}=0$ (i.e. $\hat{\mathbf{H}}_{k k}=\mathbf{H}_{k k}$ ) for all $k$, and thus $\mathbf{X}_{k k}$ is not a random variable in this case. From (9), the outage event at user $k$ is written as

$$
\mathbf{Y}_{k}=\sum_{l \neq k} \mathbf{X}_{k l}^{H} \mathbf{X}_{k l} \geq y \text {. }
$$

Let $\mathbf{X}_{k} \triangleq\left[\mathbf{X}_{k l}\right]_{l \neq k}$. Since the elements of $\mathbf{X}_{k}$ are mutually independent, the covariance matrix of $\mathbf{X}_{k}$ is $\sigma_{h}^{2} \mathbf{I}_{K-1}$ i.e., $\mathbf{R}=$ $\sigma_{h}^{2} \mathbf{I}=\boldsymbol{\Lambda}$. From (14) $\overline{\mathbf{Q}}=\mathbf{I}_{K-1}$, and thus $\mathbf{Q}=\sigma_{h}^{2} \mathbf{I}$ from (11). Substituting these variables in (12) yields

$$
\frac{1}{2 \pi} \int_{-\infty}^{\infty} \frac{e^{y(j w+\beta)}}{j w+\beta} \cdot \frac{e^{-\frac{\sigma_{h}^{2}(j w+\beta)}{1+\sigma_{h}^{2}(j w+\beta)}\|\mathbf{q}\|^{2}}}{\left(1+\sigma_{h}^{2}(j w+\beta)\right)^{K-1}} d w .
$$

Let $s=j w+\beta$ and $\mathcal{C}$ be a contour encircling the left half plane and the imaginary axis of the complex plane. Then, the integral is given by

$$
\frac{1}{2 \pi j} \int_{\beta-j \infty}^{\beta+j \infty} \frac{e^{y s}}{s} \cdot \frac{e^{-\frac{\sigma_{h}^{2} s}{1+\sigma_{h}^{2} s}\|\mathbf{q}\|^{2}}}{\left(1+\sigma_{h}^{2} s\right)^{K-1}} d s=\frac{1}{2 \pi j} \oint_{\mathcal{C}} F(s) d s
$$

${ }^{\dagger} \beta$ is chosen to be a small positive scalar such that $\mathbf{I}+\beta \mathbf{Q}$ is positive definite.
Here the definition of $F(s)$ is clear, and $F(s)$ has two singular points at $s=0$ and $-1 / \sigma_{h}^{2}$. From the residue theorem,

$$
\operatorname{Pr}\left\{\mathbf{Y}_{k} \leq y\right\}=\sum_{s=0,-1 / \sigma_{h}^{2}} \operatorname{Res} F(s) \text { for } y \geq 0 .
$$

It is obvious that $\operatorname{Res} F(0)=1$. To compute $\operatorname{Res}_{s=-\frac{1}{\sigma_{h}^{2}}} F(s)$, we introduce $G(s)$ such that

$$
\begin{aligned}
G(s) & =F\left(s-\frac{1}{\sigma_{h}^{2}}\right)=\frac{e^{y\left(s-1 / \sigma_{h}^{2}\right)}}{s-1 / \sigma_{h}^{2}} \cdot \frac{e^{-\left(1-\frac{1}{\sigma_{h}^{2} s}\right)\|\mathbf{q}\|^{2}}}{\left(\sigma_{h}^{2} s\right)^{K-1}} \\
& =e^{-\left(\frac{y}{\sigma_{h}^{2}}+\|\mathbf{q}\|^{2}\right)} \cdot \underbrace{\frac{e^{y s}}{s-1 / \sigma_{h}^{2}}}_{g(s)} \cdot \underbrace{\frac{e^{\|\mathbf{q}\|^{2} / \sigma_{h}^{2} s}}{\left(\sigma_{h}^{2} s\right)^{K-1}}}_{f(s)}
\end{aligned}
$$

Thus, the residue of $F(s)$ at $s=-1 / \sigma_{h}^{2}$ is transformed to the residue of $G(s)$ at $s=0$. Taylor and Laurent series expansions of $g(s)$ and $f(s)$ at 0 , respectively, are

$$
g(s)=\sum_{n=0}^{\infty} \frac{1}{n !} g^{(n)}(0) s^{n} \quad \text { and } \quad f(s)=\frac{1}{\left(\sigma_{h}^{2} s\right)^{K-1}} \sum_{n=0}^{\infty} \frac{1}{n !}\left(\frac{\|\mathbf{q}\|^{2}}{\sigma_{h}^{2} s}\right)^{n} .
$$

Multiplying two series and computing the coefficient of $1 / s$, we obtain the residue of $G(s)$ at zero which is given by

$$
\begin{aligned}
& \operatorname{Res}_{s=0} G(s)=\frac{1}{\left(\sigma_{h}^{2}\right)^{K-1}} e^{-\left(\frac{y}{\sigma_{h}^{2}}+\|\mathbf{q}\|^{2}\right)} \\
& \quad \times \sum_{n=K-2}^{\infty} \frac{1}{n !} g^{(n)}(0) \frac{1}{(n-K+2) !}\left(\frac{\|\mathbf{q}\|^{2}}{\sigma_{h}^{2}}\right)^{n-K+2} .
\end{aligned}
$$

Finally, $\operatorname{Pr}\{$ outage $\}=1-\operatorname{Pr}\left\{\mathbf{Y}_{k} \leq y\right\}=1-(\operatorname{Res} F(0)+$ Res $G(0))=-\operatorname{Res} G(0)$.

Next we consider the case that we have imperfect CSIT for all channels including the desired link still with one data stream per user.

Theorem 2: When only imperfect CSIT is available for all channels and $d=1$, the outage probability is given by

$$
\begin{aligned}
\operatorname{Pr}\{\text { outage }\} & =-\frac{1}{\left(\sigma_{h}^{2}\right)^{K}} e^{-\left(\frac{y}{\sigma_{h}^{2}}+\|\mathbf{q}\|^{2}\right)} \\
\times & \sum_{n=K-1}^{\infty} \frac{1}{n !} g^{(n)}(0) \frac{1}{(n-K+1) !}\left(\frac{\|\mathbf{q}\|^{2}}{\sigma_{h}^{2}}\right)^{n-K+1}
\end{aligned}
$$

$q_{k}$ is the $k$-th element of $\mathbf{q}$ defined below and $g(s)=\frac{e^{y s}}{s-1 / \sigma_{h}^{2}}$.

Proof: In this case, the outage event at user $k$ is given by

$$
\mathbf{Y}_{k}=\sum_{l=1}^{K} \mathbf{X}_{k l}^{H} \mathbf{X}_{k l} \geq y
$$

Let us redefine $\mathbf{X}_{k}:=\left[\mathbf{X}_{k l}\right]_{l \in \mathcal{K}}$. Then, the outage event is expressed with a quadratic form as $\mathbf{X}_{k}^{H} \mathbf{X}_{k} \geq \frac{\left|\mathbf{u}_{k}^{H} \hat{\mathbf{H}}_{k k} \mathbf{v}_{k}\right|^{2}}{2^{R} k-1}-\sigma^{2}$. Since the covariance matrix of $\mathbf{X}_{k}$ is $\sigma_{h}^{2} \mathbf{I}_{K}$, we have $\mathbf{Q}=$ $\sigma_{h}^{2} \mathbf{I}_{K}$ and $\mathbf{q}=\left[q_{k}\right]_{k \in \mathcal{K}}=\mathbb{E}\left\{\mathbf{X}_{k}\right\} / \sigma_{h}$. Substituting $\mathbf{Q}$ and $\mathbf{q}$ in (12) and following a similar procedure to that in the proof 
of Theorem 1, we have the closed-form expression (18) for the outage probability.

Now we consider the general case that all CSIT is imperfect and the number of data streams per user is arbitrary. The outage probability in this case is given by the following theorem.

Theorem 3: When $d \geq 2$, the outage probability is given by

$$
\begin{aligned}
& \operatorname{Pr}\{\text { outage }\}= \\
& \quad-\sum_{i=1}^{K d} \frac{e^{-\left(\frac{\sigma^{2}}{\lambda_{i}}+\left|q_{i}\right|^{2}\right)}}{\lambda_{i}} \sum_{n=0}^{\infty}\left(\frac{1}{n !}\right)^{2}\left(\frac{\left|q_{i}\right|^{2}}{\lambda_{i}}\right)^{n} g^{(n)}(0),
\end{aligned}
$$

where $q_{k}$ is the $k$-th element of $\mathbf{q}$ defined below in the proof, and

$$
g(s)=\frac{e^{y s}}{s-1 / \lambda_{i}} \cdot \frac{e^{-\sum_{l \neq i} \frac{\lambda_{l}\left(s-1 / \lambda_{i}\right)}{1+\lambda_{l}\left(s-1 / \lambda_{i}\right)}\left|q_{l}\right|^{2}}}{\prod_{l \neq i}\left(1-\lambda_{l}\left(s-1 / \lambda_{i}\right)\right)},
$$

where $\lambda_{i}$ are the eigenvalues ${ }^{\ddagger}$ of $\mathbf{R}$ also defined below in the proof.

Proof: In this case, the outage event at stream $m$ of user $k$ is given in (9). Let $\mathbf{X}_{k}^{(m)}=\left[\mathbf{X}_{k 1}^{(m 1)}, \cdots, \mathbf{X}_{k 1}^{(m d)}, \cdots, \mathbf{X}_{k K}^{(m d)}\right]^{T}$. Then, the covariance matrix of $\mathbf{X}_{k}^{(m)}$ has a block-diagonal form as $\mathbf{R}=\sigma_{h}^{2} \operatorname{diag}\left(\mathbf{R}_{i}\right)_{i \in \mathcal{K}}$ where each block is given by

$$
\mathbf{R}_{i}=\left[\begin{array}{cccc}
1 & \left(\mathbf{v}_{i}^{(2)}\right)^{H} \mathbf{v}_{i}^{(1)} & \cdots & \left(\mathbf{v}_{i}^{(d)}\right)^{H} \mathbf{v}_{i}^{(1)} \\
\left(\mathbf{v}_{i}^{(1)}\right)^{H} \mathbf{v}_{i}^{(2)} & 1 & \cdots & \left(\mathbf{v}_{i}^{(d)}\right)^{H} \mathbf{v}_{i}^{(2)} \\
\ldots & \ddots & \ddots & \vdots \\
\left(\mathbf{v}_{i}^{(1)}\right)^{H} \mathbf{v}_{i}^{(d)} & \left(\mathbf{v}_{i}^{(2)}\right)^{H} \mathbf{v}_{i}^{(d)} & \cdots & 1
\end{array}\right] .
$$

Let $\mathbf{R}=\mathbf{U} \boldsymbol{\Lambda} \mathbf{U}^{H}$ be the eigen-decomposition of $\mathbf{R}$. Then, $\mathbf{Q}=\boldsymbol{\Lambda}^{\frac{H}{2}} \mathbf{U}^{H} \overline{\mathbf{Q}} \mathbf{U} \boldsymbol{\Lambda}^{\frac{1}{2}}, \mathbf{q}=\boldsymbol{\Lambda}^{-\frac{1}{2}} \mathbf{U}^{H} \mathbb{E}\left\{\mathbf{X}_{k}^{(m)}\right\}$, where $\overline{\mathbf{Q}}=$ $\mathbf{I}_{K d}$ Substituting $\mathbf{Q}$ and $\mathbf{q}$ in (12) and following a similar procedure to those in the previous proofs, we have the closedform expression (20) for the outage probability.

\section{Outage-Constrained Beam Design for MiMO INTERFERENCE CHANNELS}

In this section, we propose an iterative beam design algorithm based on the closed-form expressions for the outage probability obtained in the previous section. Due to the structure of the problem, the simultaneous joint optimal design for both transmit and receive beam vectors is a hard problem, and thus we resort to an iterative approach to solve the weighted sum outage-rate maximization problem (5)-(7). In the proposed scheme, we initialize $\left\{\mathbf{v}_{k}^{(m)}\right\}$ and $\left\{\mathbf{u}_{k}^{(m)}\right\}$ properly, and then find optimal rate-tuple $\left(R_{1}^{(1)}, \cdots, R_{K}^{(d)}\right)$ that maximizes the weighted sum for a given $\epsilon, \hat{\mathcal{H}},\left\{\mathbf{v}_{k}^{(m)}\right\}$ and $\left\{\mathbf{u}_{k}^{(m)}\right\}$ while satisfying the outage constraint. Second, for the obtained rate-tuple and receive beam vectors $\left\{\mathbf{u}_{k}^{(m)}\right\}$ in the first step, we update the transmit beam vectors $\mathbf{v}_{k}^{(m)}$ so that they minimize the maximum outage probability among all streams and receivers. Since one transmit beamforming vector

\footnotetext{
${ }^{\ddagger}$ In Theorem 3, we assume that $\left\{\tilde{\lambda}_{i}\right\}$ are all distinct.
}

affects the outage probability of other receivers, we iterate the procedure from the first stream of transmitter 1 to the last stream of transmitter $K$ repeatedly until it converges. Next, the receive beam vectors $\left\{\mathbf{u}_{k}^{(m)}\right\}$ are determined to minimize the outage probability of stream $m$ at receiver $k$ with the obtained $\left\{\mathbf{v}_{k}^{(m)}\right\}$ and rate-tuple in the previous step similar to the previous step. Finally, with the updated transmit and receive beam vectors we recalculate the rate-tuple to maximize the sum rate satisfying the outage constraint. In this step, the outage probability for each stream is increased again up to the maximum allowed value. We iterate these steps until the sum outage-rate does not change. The proposed beam design algorithm is summarized in Table IV.

\section{Algorithm: Weighted sum outage-rate maximization with channel uncertainty}

Input: channel estimate $\hat{\mathcal{H}}$, weighting coefficients $\left(w_{1}^{(1)}, \cdots, w_{K}^{(d)}\right)$, and allowed outage probability $\epsilon$.

0 . Initialize $\left\{\mathbf{v}_{k}^{(m)}\right\}$ as an arbitrary unit vector and $\left\{\mathbf{u}_{k}^{(m)}\right\}$ properly, e.g., as the whitened matched filter.

1. For given $\left\{\mathbf{V}_{k}\right\}$ and $\left\{\mathbf{U}_{k}\right\}$, find $\left(R_{1}^{(1)}, \cdots, R_{K}^{(d)}\right)$ that maximizes $\sum_{k=1}^{K} \sum_{m=1}^{d} w_{k}^{(m)} R_{k}^{(m)}$ while the outage constraint is satisfied i.e., $\left(R_{1}^{(1)}, \cdots, R_{k}^{(d)}\right) \in \mathcal{R}_{\epsilon}\left(\mathbf{V}_{k}, \mathbf{U}_{k}, \hat{\mathcal{H}}\right)$.

2. For transmitter $k$, obtain $\mathbf{v}_{k}^{(m)}$ that minimizes the maximum outage probability among all streams for given rate-tuple $\left(R_{1}^{(1)}, \cdots, R_{K}^{(d)}\right)$ and $\left\{\mathbf{U}_{k}\right\}$.

Iterate step 2 from the first stream of transmitter 1 to the last stream of transmitter $K$ until $\left\{\mathbf{V}_{1}, \cdots, \mathbf{V}_{K}\right\}$ do not change.

3. For receiver 1 to $K$, obtain the receive filter $\mathbf{u}_{k}^{(m)}$ that minimize the outage probability of stream $m$ at receiver $k$ for $\left\{\mathbf{V}_{k}\right\}$ given from step 2 and $R_{k}^{(m)}$ from step 1 .

4. Go to 1 and repeat until converges.

Theorem 4: The proposed beam design algorithm converges.

Proof: Let $\left\{\mathbf{V}_{k}[n]\right\}$ and $\left\{\mathbf{U}_{k}[n]\right\}$ be the input of the algorithm at the $n$-th iteration. In step 1 of the algorithm, a Pareto optimal point of $\mathcal{R}_{\epsilon}\left(\mathbf{V}_{k}[n], \mathbf{U}_{k}[n], \hat{\mathcal{H}}\right)$ is achieved with the outage constraint satisfied with equality, since $\operatorname{Pr}\{\log (1+$ $\left.\left.\left.\operatorname{SINR}_{k}\right|_{\hat{\mathcal{H}}}\right) \leq R_{k}^{\prime}\right\}=\epsilon$ for some $R_{k}^{\prime}>R_{k}$ if $\operatorname{Pr}\{\log (1+$ $\left.\left.\left.\mathrm{SINR}_{k}\right|_{\hat{\mathcal{H}}}\right) \leq R_{k}\right\}<\epsilon$. In the second and third steps, the algorithm determines $\left\{\mathbf{V}_{k}[n+1]\right\}$ and $\left\{\mathbf{U}_{k}[n+1]\right\}$ that minimize the outage probability of the rate-tuple computed in step 1 . The reduced outage probability at a given rate-tuple implies that $\mathcal{R}_{\epsilon}\left(\mathbf{V}_{k}[n], \mathbf{U}_{k}[n], \hat{\mathcal{H}}\right) \subset \mathcal{R}_{\epsilon}\left(\mathbf{V}_{k}[n+1], \mathbf{U}_{k}[n+1], \hat{\mathcal{H}}\right)$, i.e., the rate region expands; at the first step of the $(n+1)$ st iteration, an enhanced rate-tuple is obtained and the sum outage-rate increases monotonically. Since the outage rate is upper bounded by the interference-free capacity which is finite, the proposed beam design algorithm converges by the monotone convergence theorem.

The convergence of the algorithm does not guarantee that the proposed beam design algorithm achieves the optimal Pareto point of $\mathcal{R}_{\epsilon}(\hat{\mathcal{H}})$ which is the global optimal, but the algorithm converges to a local optimal point at least. 


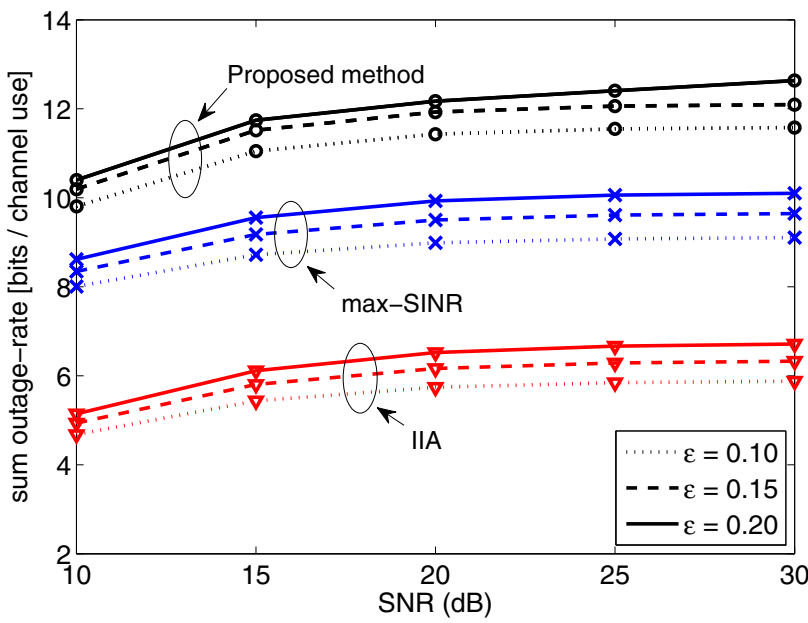

Fig. 1. Weighted sum outage-rate when $\mathbf{w}=[1,1,1]^{T}$ and $\epsilon=0.10,0.15$, and 0.20 .

\section{Numerical Results}

To evaluate the performance of the proposed design method we considered the simple case of $K=3, d=1$ and perfect CSIT for the desired links. The elements of channel estimates $\hat{\mathbf{H}}_{k l}$ were drawn from $\mathcal{N}(0,1)$ and the uncertainty $\mathbf{E}_{k l}$ with distribution $\mathcal{N}\left(0, \sigma_{h}^{2}\right)$ was added to $\hat{\mathbf{H}}_{k l}$ to produce the true channel value. The IIA and max-SINR algorithms in [4] were adopted as the reference beam design. Although the two algorithms were originally proposed to design beam vectors with perfect channel information, we applied the two algorithms to design beam vectors by treating imperfect channel $\hat{\mathcal{H}}$ as true channel.

Fig. 1 shows the weighed sum outage-rate when $\sigma_{h}^{2}=0.05$, $\mathbf{w}=[1,1,1]^{T}$, and several $\epsilon$. The $\epsilon$-outage data-rate of maxSINR and IIA algorithms is defined as the maximum data rate that can be achieved under the outage constraint of $\epsilon$ using the beams designed by the max-SINR and IIA algorithms. Note from Theorem 1 that the outage event at user $k$ occurs if

$$
\sum_{l \neq k} \mathbf{X}_{k l}^{H} \mathbf{X}_{k l} \geq \frac{\left|\mathbf{u}_{k}^{H} \mathbf{H}_{k k} \mathbf{v}_{k}\right|^{2}}{2^{R_{k}}-1}-\sigma^{2},
$$

where $\mathbf{X}_{k l}=\mathbf{u}_{k}^{H}\left(\hat{\mathbf{H}}_{k l}+\mathbf{E}_{k l}\right) \mathbf{v}_{l}$. When $\left\{\mathbf{u}_{k}\right\}$ and $\left\{\mathbf{v}_{k}\right\}$ are given, the outage probability is determined from (13) as a function of the target rate $R_{k}$. Thus, for the beam vectors designed by the max-SINR and IIA algorithms, $\epsilon$-outage datarate $R_{k}$ is easily obtained.

Fig. 1 shows that the proposed method outperforms the other two methods in the outage-rate as expected, and the sum outage-rate increases as $\epsilon$ increases. The proposed method yields a sum outage-rate gain over the max-SINR algorithm of approximately $25 \%$, and a larger gain is obtained over the IIA for the same outage probability. In the case of $\sigma_{h}^{2}=0.1 \mathrm{a}$ similar result was obtained with an approximately $30 \%$ sumrate gain over the max-SINR algorithm.

\section{CONCLUSION}

In this paper, we have considered the beam design problem for maximizing the weighted sum outage-rate in MIMO interference channels when transmitters and receivers have imperfect channel information. Closed-form expressions for the outage probability have been derived for several scenarios, and an iterative beam design algorithm for maximizing the weighted sum outage-rate has been proposed based on the derived outage-probability expression. Simulations show that the proposed algorithm yields a significant gain in the outagerate compared with the previous beam design methods in MIMO interference channels which are suboptimal in the outage sense.

\section{REFERENCES}

[1] V. R. Cadambe and S. A. Jafar, "Interference alignment and degrees of freedom of the $K$-user interference channel," IEEE Trans. Inf. Theory, vol. 54, pp. 3425 - 3441, Aug. 2008.

[2] T. Gou and S. A. Jafar, "Degrees of freedom of the $K$ user $M \times$ $N$ MIMO interference channel," ArXiv pre-print cs.IT/0809.0099, Aug. 2008.

[3] C. M. Yetis, T. Gou, S. A. Jafar, and A. H. Kayran, "On feasibility of interference alignment in MIMO interference netowrks," IEEE Trans. Signal Process., vol. 58, pp. 4771 - 4782, Sep. 2010.

[4] K. Gomadam, V. R. Cadambe, and S. A. Jafar, "Approaching the capacity of wireless networks through distributed interfernce alignment," ArXiv pre-print cs.IT/0803.3816, Mar. 2008.

[5] I. Santamaria, O. Gonzalez, R. H. Jr., and S. W. Peters, "Maximum sumrate interference alignment algorithms for MIMO channels ," in Proc. of Globecom, (Miami, FL), Dec. 2010.

[6] D. Schmidt, S. Changxin, R. Berry, M. Honig, and W. Utschick, "Minimum mean squared error interference alignment," in Proc. of Asilomar, (Pacific Grove, CA), Nov. 2009.

[7] S. W. Peters and R. W. Heath, "Interference alignment via alternating minimization," in Proc. of ICASSP, (Taipei, Taiwan), Apr. 2009.

[8] H. Yu and Y. Sung, "Least squares approach to joint beam design for interference alignment in multiuser multi-input multi-output interference channels," IEEE Trans. Signal Process., vol. 58, pp. 4960 - 4966, Sep. 2010.

[9] M. Vu and A. Paulraj, "MIMO wireless linear precoding," IEEE Signal Processing Mag., vol. 24, pp. 86 - 105, Sep. 2007.

[10] E. Chiu, V. Lau, H. Huang, T. Wu, and S. Liu, "Robust Transceiver Design for $K$-Pairs Quasi-Static MIMO Interference Channels via SemiDefinite Relaxation ," IEEE Trans. Wireless Commun., vol. 9, pp. 3762 - 3769, Dec. 2010.

[11] H. Shen, B. Li, M. Tao, and X. Wang, "MSE-based transceiver designs for the MIMO interference channel," IEEE Trans. Wireless Commun., vol. 9, pp. 3480-3489, Nov. 2010.

[12] C. Wang, E. K. S. Au, R. D. Murch, W. H. Mow, R. S. Cheng, and V. Lau, "On the performance of MIMO zero-forcing receiver in the presence of channel estimation error," IEEE Trans. Wireless Commun. vol. 6, pp. 805 - 810, Mar. 2007.

[13] J. Lindblom, E. Karipidis, and E. Larsson, " Outage rate regions for the MISO IFC ," in Proc. of Asilomar, (Pacific Glove, CA.), Nov. 2009.

[14] J. Lindblom, E. Larsson, and E. Jorswieck, "Parametrization of the MISO IFC rate region: The case of partial channel state information," IEEE Trans. Wireless Commun., vol. 9, pp. 500 - 504, Feb. 2010.

[15] J. Gurland, "Distribution of definite and of indefinite quadratic forms," Annals of Mathematical Statistics, vol. 26, pp. 122-127, 1955.

[16] D. Raphaeli, "Distribution of noncentral quadratic forms in complex normal variables ," IEEE Trans. Inf. Theory, vol. 42, pp. 1002 - 1007 , May 1996.

[17] T. Al-Naffouri and B. Hassibi, "On the distribution of indefinite quadratic forms in Gaussian random varables," in Proc. of ISIT, (Seoul, Korea), Jun.-Jul. 2009. 